\title{
Medicinal plants used by "Passo da Ilha" rural community in the city of Pato Branco, southern Brazil
}

\author{
JOSÉ A. MARCHESE ${ }^{1}$, LIN C. MING ${ }^{2}$, LUCIA DE FRANCESCHI ${ }^{1}$, RUBIA C. CAMOCHENA ${ }^{1}$, \\ GREICE D.R. GOMES ${ }^{1}$, MARCOS V. PALADINI ${ }^{1}$, DIOGO CAPELIN ${ }^{1}$ and CRISTINE F. MARCHESE ${ }^{3}$ \\ ${ }^{1}$ Curso de Agronomia, Universidade Tecnológica Federal do Paraná, Via do Conhecimento, km 1 \\ 85503-390 Pato Branco, PR, Brasil \\ ${ }^{2}$ Faculdade de Ciências Agrárias, Universidade Estadual Paulista, Rua José Barbosa de Barros, 1780 \\ Fazenda Lageado, 18603-970 Botucatu, SP, Brasil \\ ${ }^{3}$ Secretaria de Educação, Prefeitura Municipal de Pato Branco, Rua Caramuru, 271 \\ Centro, 85501-060 Pato Branco, PR, Brasil \\ Manuscript received on June 13, 2008; accepted for publication on June 4, 2009; \\ presented by AleXANDER W.A. KellneR
}

\begin{abstract}
The scope of this work was to rescue and document the traditional knowledge regarding the medicinal plants used by Passo da Ilha rural community, in Pato Branco, Paraná State, Southern Brazil $\left(26^{\circ} 11^{\prime} \mathrm{S}, 52^{\circ} 36^{\prime} \mathrm{W}\right.$ and $760 \mathrm{~m}$ high). Structured interviews were made in field research with 16 informants who had the traditional knowledge about medicinal plants. The research was carried out from October to December 2000. The plants were collected in the field, identified and their vouchers were housed at the Herbarium "Irina Delanova De Gemtchjnicov" (BOTU) of São Paulo State University, in Botucatu. A total of 47 botanical families and 114 species of medicinal plants were identified. These plants were suitable for more than 30 different medicinal uses. The residents are mainly of European descent, which justify the presence of many exotic plants. The knowledge on how to use medicinal plants properly is held mainly by the females, and decreases in the newer generations, denoting "cultural erosion" in progress.
\end{abstract}

Key words: cultural erosion, ethnobotany, medicinal plants, traditional knowledge.

\section{INTRODUCTION}

The use of medicinal plants and their derived forms have produced the basis of the therapeutics through the centuries. With the Chemistry evolution, starting from the $19^{\text {th }}$ century, the way in which plants were used changed: from the direct use of these plants alone or mixed to the artificial reproduction of isolated active substances through active molecules. As a consequence, the plants that form these substances were considered to be less important.

Therefore, the relative knowledge of the medicinal plants is being lost through the generations. Despite

Correspondence to: José Abramo Marchese

E-mail: abramo@pq.cnpq.br of this, most of the world's population has little access to conventional medical care - which relies on the prescription of synthetic medication that is usually too expensive for them. In this sense, a considerable additional difficulty is the limited availability of synthetic medications for the poorer populations in the government health service. One of the viable and inexpensive alternatives to access medication would be the use of medicinal plants and their mixtures by the communities as a complementary form of therapeutics. Most populations present high cultural attachment to their own traditions, a factor that would facilitate the development of a community program that uses medicinal plants with proven therapeutic action. It could encourage the tradi- 
tional uses of the plants in the communities (Farnsworth et al. 1985, Farnsworth 1988).

According to Farnsworth et al. (1985), and Elizabetsky (1991, 2000), a fairly high percentage of useful plant-derived drugs was discovered as a result of scientific research of well-known plants used in traditional medicine, and the authors have concluded that this is a good approach for discovering other useful drugs derived from plants.

The scope of this work was to rescue and document the traditional knowledge regarding the medicinal plants used by the population of the rural community called "Passo da Ilha" in Pato Branco, Paraná State, Brazil.

\section{MATERIALS AND METHODS}

"Passo da Ilha" rural community in Pato Branco, Paraná State, Brazil, $\left(26^{\circ} 11^{\prime} \mathrm{S} ; 52^{\circ} 36^{\prime} \mathrm{W}\right)$ (Fig. 1), is mainly composed by small properties where subsistence agriculture is practiced. The first objective of this work was to have discussions in informal meetings with the community, so that they understand and allow the activities that would be carried out. The ethnobotanical survey was conducted by structured interviews with 16 informants who had the knowledge of medicinal plants usage in their families (Martin 1995, Alexiades 1996). The interviews were carried out from October to December 2000.

The plants were collected in field, pressed and labeled (Martin 1995, Ming 1995, Alexiades 1996). Plant species were identified and voucher specimens housed at the Herbarium "Irina Delanova De Gemtchjnicov" (BOTU) of São Paulo State University in Botucatu. For protection purposes, regarding the genetic resources and the traditional knowledge of medicinal use of these plants by the community, no information related to medicinal use was reported. This publication option is in agreement with other researcher's thoughts (Laird and Kate 2002) and we found it to be the more ethical form to communicate the results in ethnobotanical survey.

\section{RESULTS AND DISCUSSION}

A total of 47 botanical families and 114 species of medicinal plants were identified; the prevalent fam- ilies were Asteraceae and Lamiaceae, with 20 and 16 species, respectively (Table I). These data agree with those of Dorigoni et al. (2001) and Garlet and Irgang (2001), in ethnobotanical studies that were carried out in the South of Brazil, where the dominant species was Asteracea, followed by Lamiaceae. The high number of species mentioned for medicinal use is in contrast with the few informants interviewed (16), which shows a great knowledge of medicinal plants mainly by elderly people. These numbers reflect the value of the traditional knowledge kept by the elderly people from communities and their importance to both cultural and biological conservation (Heinrich et al. 1998, Amorozo 2002, Monteiro et al. 2006).

The medicinal plants were suitable for more than 30 different medicinal uses, predominantly for respiratory diseases and stomachic diseases, with 17.22\% (57 citations) and 9.97\% (33 citations), respectively (Table II). These data agree with those of Garlet and Irgang (2001) and Marodin and Baptista (2001) who found similar results in ethnobotanical studies that were carried out in the South of Brazil and in the West of Brazil by Amorozo (2002), where respiratory and stomachic diseases are the two main health problems. The cold and wet weather in winter could worsen the health conditions of the inhabitants in most parts of Paraná State and affect their respiratory conditions.

Motomiya et al. (2004), while investigating the use of medicinal plants in Cassilândia - Mato Grosso, Brazil, noticed that the most frequently mentioned plants are those used for stomach, intestine and breathing illness treatment such as flu, bronchitis and cough. Franco and Barros (2006) found that the greatest variety of different medicinal plants was for the control of breathing illnesses, coming to $26.7 \%$, in Esperantina Piauí, Brazil.

Figure 2 shows that many medicinal species were recurrently mentioned by different informants possibly due to the fact that they are long-standing inhabitants of the locations (Table III) and exchange plants and information. A great number of exotic plants were listed in the interviews (Fig. 2), and this is probably due to the fact that the informants have predominantly European ancestry (Italian 38\%; German 13\%; Portuguese 9\%; Ukrainian $6 \%$; Poles $3 \%$ ) and only $31 \%$ of Brazilian 
BRAZIL

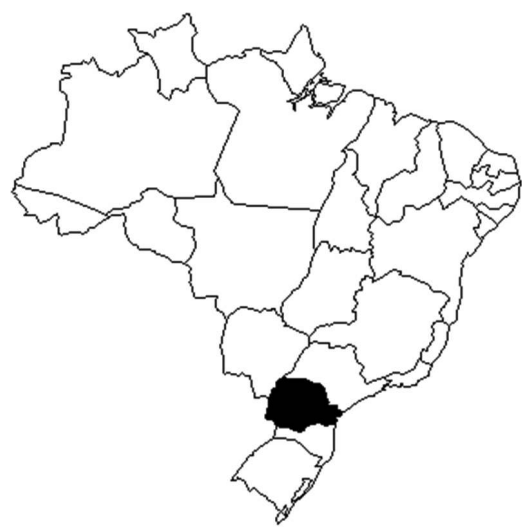

Paraná state

\section{PARANÁ STATE}

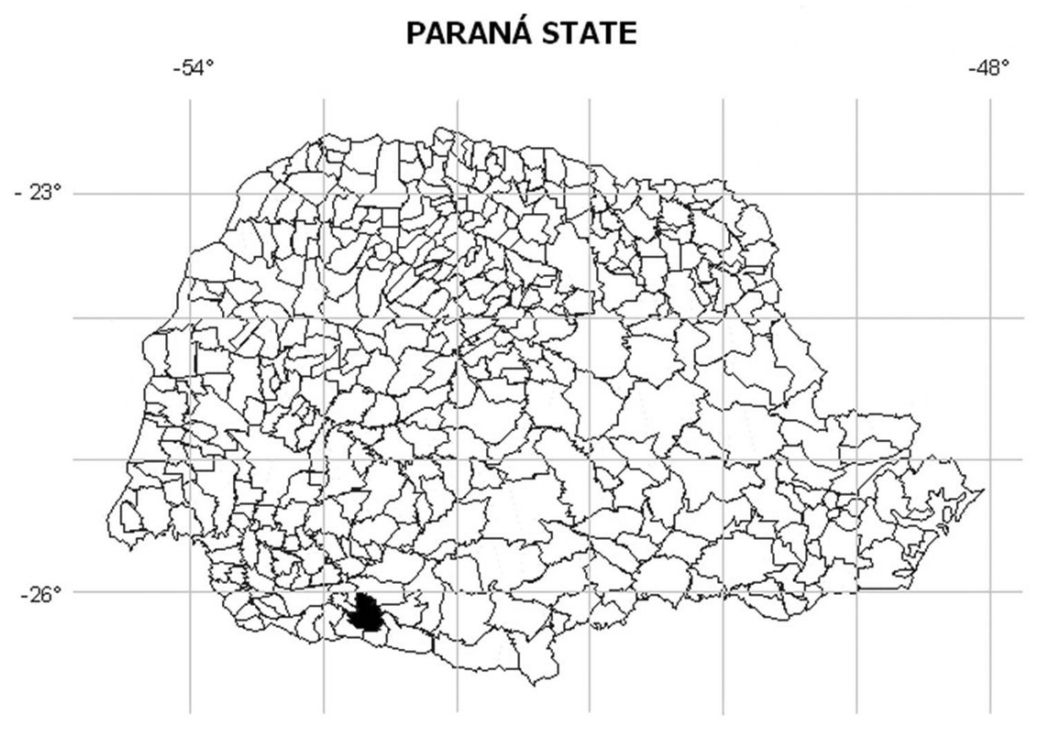

Pato Branco

Fig. 1 - Geographical location of Pato Branco-PR, Southern Brazil $\left(26^{\circ} 11^{\prime} \mathrm{S} ; 52^{\circ} 36^{\prime} \mathrm{W}\right)$.

TABLE I

Botanical families and the number of species identified during the ethnobotanical survey carried out in "Passo da Ilha" rural community, in the city of Pato Branco, Southern Brazil.

\begin{tabular}{lccc|lc}
\hline Botanical families & ${ }^{1}$ spp. & Botanical families & ${ }^{1}$ spp. & Botanical families & ${ }^{1}$ spp. \\
\hline Agavaceae & 1 & Cucurbitaceae & 1 & Plantaginaceae & 2 \\
\hline Alismataceae & 1 & Equisetaceae & 1 & Poaceae & 2 \\
\hline Aloeaceae & 1 & Euphorbiaceae & 2 & Polygonaceae & 1 \\
\hline Amaranthaceae & 2 & Fabaceae & 4 & Polypodiaceae & 1 \\
\hline Apiaceae & 3 & Lamiaceae & 16 & Pteridaceae & 2 \\
\hline Aristolochiaceae & 1 & Lauraceae & 2 & Punicaceae & 1 \\
\hline Asteraceae & 20 & Liliaceae & 3 & Rosaceae & 6 \\
\hline Bignoniaceae & 1 & Lythraceae & 1 & Rutaceae & 1 \\
\hline Boraginaceae & 1 & Malvaceae & 2 & Simaroubaceae & 1 \\
\hline Brassicaceae & 3 & Mimosaceae & 1 & Solanaceae & 2 \\
\hline Caesalpinaceae & 3 & Moraceae & 1 & Urticaceae & 1 \\
\hline Caprifoliaceae & 1 & Myrtaceae & 5 & Verbenaceae & 4 \\
\hline Celastraceae & 2 & Papaveraceae & 1 & Violaceae & 1 \\
\hline Chenopodiaceae & 1 & Passifloraceae & 1 & Vitaceae & 1 \\
\hline Commelinaceae & 1 & Phytolaccaceae & 1 & Zingiberaceae & 2 \\
\hline Crassulaceae & 1 & Piperaceae & 1 & & \\
\hline \multicolumn{2}{l}{} & 1 Number & different species found per botanical family &
\end{tabular}

ancestry. Paraná State is one of the Brazilian States that has received waves of European immigrants since end of $19^{\text {th }}$ century, which contributes to its great cultural diversity. In addition, 65 exotic species and 49 native species of medicinal plants were identified (Appendix
I). These data are similar to those observed by Dorigoni et al. (2001), Garlet and Irgang (2001) and Marodin and Baptista (2001) in ethnobotanical studies carried out in the south of Brazil, showing the great influence of the European immigrants. 
TABLE II

Number and percentage of citations of the ethnotherapeutic uses by different informants during the ethnobotanical survey carried out in "Passo da Ilha" rural community, in the city of Pato Branco, Southern Brazil.

\begin{tabular}{l|c|c}
\hline Ethnotherapeutic uses & $\begin{array}{c}\text { Number of recurrently } \\
\text { mentioned species }\end{array}$ & $\begin{array}{c}\text { Percentage of recurrently } \\
\text { mentioned species }\end{array}$ \\
\hline Hypertension & 6 & 1.81 \\
\hline Heart diseases & 6 & 1.81 \\
\hline Urinary bladder diseases & 7 & 2.11 \\
\hline Worms & 8 & 2.11 \\
\hline Pains in the body & 9 & 2.42 \\
\hline Anxiety & 9 & 2.72 \\
\hline Fever & 9 & 2.72 \\
\hline Hepatitis & 11 & 2.72 \\
\hline Migraine & 12 & 3.32 \\
\hline Infections & 14 & 3.63 \\
\hline Bruise & 14 & 4.23 \\
\hline Feminine diseases & 16 & 4.23 \\
\hline Renal diseases & 26 & 4.83 \\
\hline Intestinal diseases & 32 & 7.85 \\
\hline Blood diseases & 33 & 9.67 \\
\hline Stomachic diseases & 57 & 9.97 \\
\hline Respiratory diseases & 331 & 17.22 \\
\hline Anothers (less than five & & \\
times mentioned) & 75.62 \\
\hline Total & 7500 \\
\hline
\end{tabular}

TABLE III

The informants' gender and time of living in "Passo da Ilha" rural community, in the city of Pato Branco, Southern Brazil.

\begin{tabular}{|c|c|c|c|c|c|}
\hline Informants & Gender & $\begin{array}{l}\text { Amount of years } \\
\text { living in the place }\end{array}$ & Informants & Gender & $\begin{array}{l}\text { Amount of years } \\
\text { living in the place }\end{array}$ \\
\hline 1 & q & 23 & 9 & q & 25 \\
\hline 2 & Q & 23 & 10 & $\sigma^{x}$ & 23 \\
\hline 3 & q & 20 & 11 & q & 27 \\
\hline 4 & q & 24 & 12 & q & 64 \\
\hline 5 & q & 15 & 13 & q & 44 \\
\hline 6 & q & 20 & 14 & q & 65 \\
\hline 7 & $\sigma^{\pi}$ & 20 & 15 & q & 30 \\
\hline 8 & 우 & 27 & 16 & 우 & 37 \\
\hline
\end{tabular}

$q=$ feminine; $\sigma^{\top}=$ masculine 


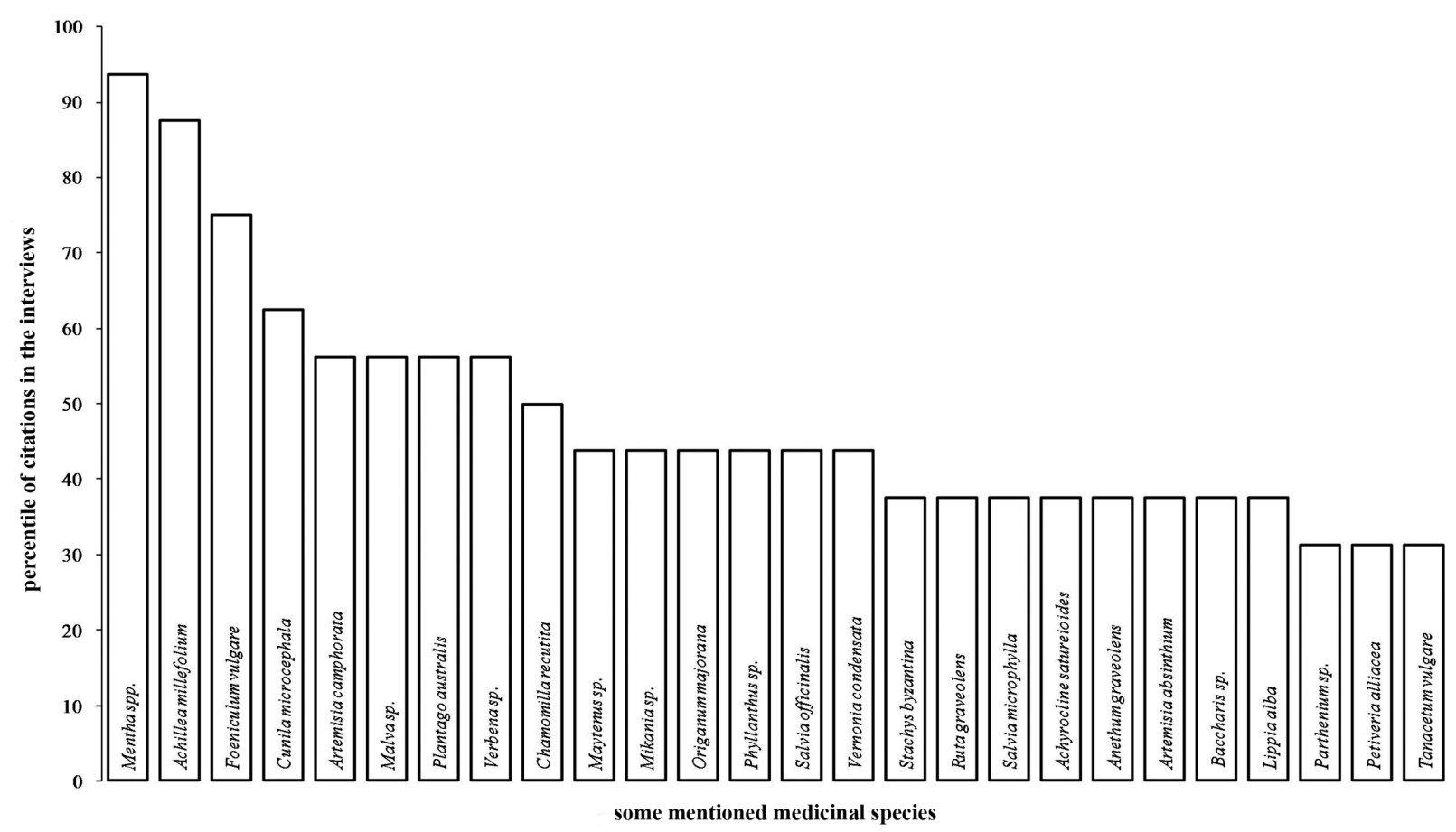

Fig. 2 - Percentages of recurrently mentioned species from different informants during the ethnobotanical survey carried out in "Passo da Ilha" rural community, in the city of Pato Branco, Southern Brazil.

Until $19^{\text {th }}$ century the inhabitants of Paraná were indigenous and belonged to the Xetá, Guarani and Kaingang ethnic groups. The availability of land possessing one of the biggest reserves of Araucaria angustifolia (Brazilian pine) in Brazil and the regime of free properties attracted the immigrants to the region of Pato Branco (D.T.P. Menosso, unpublished data). It has been known that, between 1903 and 1910, there was the first settling of European immigrants in Pato Branco (Voltolini 1996).

The question of gender related to the sharing of knowledge shows that the transfer of knowledge through the generations about the use of medicinal plants is of women's responsibility. As a result, it is the mothers and grandmothers' duty to look for some therapeutical resource when a relative is ill (Chrisman 1977).

According to Davim et al. (2003), women have been considered the custodians of the family in a dedicated and caring manner for years, assuring good health and a quick recovery in the event of an illness. They have inherited this cultural habit from their ancestors, which results in a sensitive empirical knowledge accompanied by some intuition.
As reported by Monteiro et al. (2006), the differences in knowledge related to gender can be affected by cultural oscillations concerning factors such as the nonhomogeneous distribution of medicinal plant knowledge and the generally small native population.

However, in Figure 3 a clear tendency of erosion of this knowledge is noticed, as the youngest informants mentioned a lower number of plants than the older ones. This tendency shows the need of works about recovery and practice of the knowledge of medicinal plants, clearly important in communities that lack therapeutic resources for the combat of illnesses.

Some authors have discussed this issue (Hanazaki et al. 2000, Begossi et al. 2002, Amorozo 2004), and the loss of that knowledge could be associated with the cultural disconnection of the generations.

All information found in this research, backed by the scientific knowledge, was returned to the community through educational programs. When the ethnobotanical research was finished, a second study to define the profile of consumers of spices and medicinal plants in retail in the city of Pato Branco was carried out (Marchese et al. 2004). After the definition of the 
APPENDIX I

List of medicinal plants used in "Passo da Ilha" rural community, in the city of Pato Branco, Southern Brazil. $\mathrm{MF}=$ mentioning frequency; $\mathrm{N}=$ native; $\mathrm{E}=$ exotic; $\mathrm{HB}=$ herbaceous; $\mathrm{SB}=$ shrub; $\mathrm{SS}=$ subshrub;

$\mathbf{A R}=$ arboreous $\mathbf{E P}=$ epiphyte $\mathbf{P C}=$ plant creeper; $\mathbf{A}=$ annual $; \mathbf{B}=$ biennial; $\mathbf{P}=$ perennial.

\begin{tabular}{|c|c|c|c|c|c|}
\hline Botanical name & Common name & Voucher \# & $\mathrm{MF}$ & $\mathrm{N} / \mathrm{E}$ & Growing habit \\
\hline \multicolumn{6}{|l|}{ Agavaceae } \\
\hline Sansevieria zeylanica Willd. & espada-de-são jorge & 24029 & 1 & $\mathrm{E}$ & $\mathrm{HB} / \mathrm{P}$ \\
\hline \multicolumn{6}{|l|}{ Alismataceae } \\
\hline $\begin{array}{l}\text { Echinodorus grandiflorus } \\
\text { (Cham. and Schltdl.) Micheli }\end{array}$ & chapéu-de-couro & 24030 & 3 & $\mathrm{~N}$ & $\mathrm{HB} / \mathrm{P}$ \\
\hline \multicolumn{6}{|l|}{ Aloaceae } \\
\hline Aloe sp. & babosa fininha & 24031 & 4 & $\mathrm{E}$ & $\mathrm{HB} / \mathrm{P}$ \\
\hline \multicolumn{6}{|l|}{ Amaranthaceae } \\
\hline Alternanthera brasiliana (L.) Kuntze & terramicina; penicilina & 24032 & 6 & $\mathrm{~N}$ & $\mathrm{HB} / \mathrm{P}$ \\
\hline Gomphrena celosioides Mart. & perpétua & 24033 & 1 & $\mathrm{~N}$ & $\mathrm{HB}$ or $\mathrm{SS} / \mathrm{P}$ \\
\hline \multicolumn{6}{|l|}{ Apiaceae } \\
\hline Anethum graveolens $\mathrm{L}$. & endro; aipo; salsão; aipão & 24034 & 6 & E & $\mathrm{HB} / \mathrm{A}$ \\
\hline Foeniculum vulgare Mill. & endro; funcho & 24035 & 12 & $\mathrm{E}$ & $\mathrm{HB} / \mathrm{P}$ \\
\hline \multicolumn{6}{|l|}{ Petroselinum crispum (Mill.) } \\
\hline \multicolumn{6}{|l|}{ Aristolochiaceae } \\
\hline Aristolochia triangularis Cham. & cipó-mil-homem; mil-homem & 24037 & 3 & $\mathrm{~N}$ & $\mathrm{PC} / \mathrm{HB} / \mathrm{P}$ \\
\hline \multicolumn{6}{|l|}{ Asteraceae } \\
\hline Achillea millefolium $\mathrm{L}$. & pronto-alívio; mil-folhas; novalgina & 24038 & 14 & $\mathrm{E}$ & $\mathrm{HB} / \mathrm{P}$ \\
\hline Achyrocline satureioides (Lam.) DC. & marcela & 24039 & 6 & $\mathrm{~N}$ & $\mathrm{HB} / \mathrm{P}$ \\
\hline Arctium minus (Hill) Bernh. & bardana; terramicina & 24040 & 1 & E & $\mathrm{SB} / \mathrm{B}$ \\
\hline Artemisia absinthium $\mathrm{L}$. & losna & 24041 & 6 & $\mathrm{E}$ & $\mathrm{SS} / \mathrm{P}$ \\
\hline Artemisia camphorata Vill. & cânfora & 24042 & 9 & $\mathrm{E}$ & $\mathrm{SS} / \mathrm{P}$ \\
\hline Artemisia vulgaris $\mathrm{L}$. & artemísia & 24043 & 2 & $\mathrm{E}$ & $\mathrm{HB} / \mathrm{P}$ \\
\hline Baccharis articulata (Lam.) Pers & carqueja & 24044 & 6 & $\mathrm{~N}$ & SS/P \\
\hline Bidens pilosa $\mathrm{L}$. & picão-preto & 24045 & 3 & $\mathrm{~N}$ & $\mathrm{HB} / \mathrm{A}$ \\
\hline Calendula officinalis $\mathrm{L}$. & calêndula & 24046 & 1 & $\mathrm{E}$ & $\mathrm{HB} / \mathrm{A}$ or $\mathrm{B}$ \\
\hline Chamomilla recutita (L.) Rauschert & camomila; maçanilha & 24047 & 8 & $\mathrm{E}$ & $\mathrm{HB} / \mathrm{A}$ \\
\hline Cynara scolymus $\mathrm{L}$. & alcachofra & 24048 & 4 & $\mathrm{E}$ & $\mathrm{HB} / \mathrm{P}$ \\
\hline Elephantopus mollis Kunth & suçuaia & 24049 & 3 & $\mathrm{~N}$ & $\mathrm{HB} / \mathrm{P}$ \\
\hline Gochnatia polymorpha (Less.) Cabr. & cambará & 24050 & 1 & $\mathrm{~N}$ & $\mathrm{AR} / \mathrm{P}$ \\
\hline Mikania glomerata Spreng. & guaco & 24051 & 7 & $\mathrm{~N}$ & $\mathrm{PC} / \mathrm{P}$ \\
\hline Parthenium hysterophorus L. & artemísia; crisântemo & 24052 & 5 & $\mathrm{E}$ & $\mathrm{HB} / \mathrm{A}$ or $\mathrm{P}$ \\
\hline Senecio brasiliensis Less. & maria-mole & 24053 & 1 & $\mathrm{~N}$ & $\mathrm{HB} / \mathrm{P}$ \\
\hline Tanacetum vulgare $\mathrm{L}$. & catinga-de-mulata; artemisia & 24054 & 5 & $\mathrm{E}$ & $\mathrm{SS} / \mathrm{P}$ \\
\hline Taraxacum officinale Weber & pesacam, dente-de-leão & 24055 & 1 & $\mathrm{E}$ & $\mathrm{HB} / \mathrm{A}$ or $\mathrm{P}$ \\
\hline Vernonia condensata Baker & figatil & 24056 & 7 & $\mathrm{E}$ & $\mathrm{SB} / \mathrm{P}$ \\
\hline Wedelia paludosa $\mathrm{DC}$. & amarelinho & 24057 & 1 & $\mathrm{~N}$ & $\mathrm{HB} / \mathrm{P}$ \\
\hline \multicolumn{6}{|l|}{ Bignoneaceae } \\
\hline Jacaranda sp. & carova; paineira & 24058 & 2 & $\mathrm{~N}$ & $\mathrm{AR} / \mathrm{P}$ \\
\hline \multicolumn{6}{|l|}{ Boraginaceae } \\
\hline Symphytum officinale L. & confrei & 24059 & 2 & $\mathrm{E}$ & $\mathrm{HB} / \mathrm{P}$ \\
\hline \multicolumn{6}{|l|}{ Brassicaceae } \\
\hline Brassica oleracea var. acephala & couve & 24060 & 1 & $\mathrm{E}$ & $\mathrm{HB} / \mathrm{A}$ \\
\hline Coronopus didymus (L.) Smith. & mentruz & 24061 & 3 & $\mathrm{E}$ & $\mathrm{HB} / \mathrm{A}$ \\
\hline Nasturtium officinale $\mathrm{R} . \mathrm{Br}$. & agrião & 24062 & 3 & $\mathrm{E}$ & $\mathrm{HB} / \mathrm{P}$ \\
\hline
\end{tabular}


APPENDIX I (continuation)

\begin{tabular}{|c|c|c|c|c|c|}
\hline Botanical name & Common name & Voucher \# & MF & $\mathrm{N} / \mathrm{E}$ & Growing habit \\
\hline \multicolumn{6}{|l|}{ Caesalpiniaceae } \\
\hline Bauhinia forficata Link. & pata-de-vaca & 24063 & 1 & $\mathrm{~N}$ & $\mathrm{AR} / \mathrm{P}$ \\
\hline Bauhinia microstachia (Raddi.) Macbr. & cipó-escada & 24064 & 1 & $\mathrm{~N}$ & $\mathrm{PC} / \mathrm{P}$ \\
\hline Senna sp. & sene & 24065 & 1 & $\mathrm{~N}$ & $\mathrm{SB} / \mathrm{P}$ \\
\hline \multicolumn{6}{|l|}{ Caprifoliaceae } \\
\hline Sambucus australis Cham. and Schltdl. & sabugueiro & 24066 & 1 & $\mathrm{~N}$ & $\mathrm{AR} / \mathrm{P}$ \\
\hline \multicolumn{6}{|l|}{ Celastraceae } \\
\hline Maythenus aquifolium Mart. & cancorosa & 24067 & 5 & $\mathrm{~N}$ & $\mathrm{AR} / \mathrm{P}$ \\
\hline Maythenus ilicifolia Reissek & espinheira-santa & 24068 & 2 & $\mathrm{~N}$ & $\mathrm{AR} / \mathrm{P}$ \\
\hline \multicolumn{6}{|l|}{ Chenopodiaceae } \\
\hline Chenopodium ambrosioides $\mathrm{L}$. & santa-maria & 24069 & 2 & $\mathrm{~N}$ & $\mathrm{HB} / \mathrm{P}$ or $\mathrm{A}$ \\
\hline \multicolumn{6}{|l|}{ Comelinaceae } \\
\hline Tradescantia elongata Meyer & troperova & 24070 & 1 & $\mathrm{~N}$ & $\mathrm{HB} / \mathrm{P}$ \\
\hline \multicolumn{6}{|l|}{ Crassulaceae } \\
\hline Bryophyllum pinnatum (Lam.) Oken & folha-gorda; folha-da-fortuna & 24071 & 1 & $\mathrm{~N}$ & $\mathrm{HB} / \mathrm{P}$ \\
\hline \multicolumn{6}{|l|}{ Cucurbitaceae } \\
\hline Cayaponia tayuya Vell. & taiuiá & 24072 & 1 & $\mathrm{~N}$ & $\mathrm{PC} / \mathrm{P}$ \\
\hline \multicolumn{6}{|l|}{ Equisetaceae } \\
\hline Equisetum pyramidale Goldn. & cavalinha & 24073 & 1 & $\mathrm{~N}$ & $\mathrm{SS} / \mathrm{P}$ \\
\hline \multicolumn{6}{|l|}{ Euphorbiaceae } \\
\hline Euphorbia prostrata Ait. & quebra-pedra-rasteiro & 24074 & 1 & $\mathrm{E}$ & $\mathrm{HB} / \mathrm{A}$ \\
\hline Phyllanthus $s p$. & quebra-pedra & 24075 & 7 & $\mathrm{~N}$ & $\mathrm{HB} / \mathrm{P}$ \\
\hline \multicolumn{6}{|l|}{ Fabaceae } \\
\hline Acacia plumosa Lowe & unha-de-gato & 24076 & 3 & $\mathrm{~N}$ & $\mathrm{SB}-\mathrm{PC} / \mathrm{P}$ \\
\hline Dalbergia sp. & gabriúva & 24077 & 1 & $\mathrm{~N}$ & $\mathrm{AR} / \mathrm{P}$ \\
\hline Desmodium adscendens (Sw.) DC. & pega-pega & 24078 & 1 & $\mathrm{~N}$ & $\mathrm{HB} / \mathrm{P}$ \\
\hline Medicago sativa L. & alfafa & 24079 & 1 & $\mathrm{E}$ & $\mathrm{HB} / \mathrm{P}$ \\
\hline \multicolumn{6}{|l|}{ Lamiaceae } \\
\hline Cunila microcephala Benth. & poejo & 24080 & 10 & $\mathrm{~N}$ & $\mathrm{HB} / \mathrm{P}$ \\
\hline Lavandula angustifolia Mill. & alfazema; osmarim & 24081 & 2 & $\mathrm{E}$ & $\mathrm{SS} / \mathrm{P}$ \\
\hline Leonotis nepetaefolia (L.) R.Br. & cordão-de-frade & 24082 & 1 & $\mathrm{E}$ & HB-SS/A \\
\hline Leonurus sibiricus L. & arnica & 24083 & 2 & $\mathrm{E}$ & $\mathrm{HB} / \mathrm{A}$ or $\mathrm{B}$ \\
\hline Melissa officinalis L. & cidreira; melissa & 24084 & 3 & $\mathrm{E}$ & $\mathrm{HB} / \mathrm{P}$ \\
\hline Mentha citrata Ehrh. & alevante & 24085 & 1 & $\mathrm{E}$ & $\mathrm{HB} / \mathrm{P}$ \\
\hline Mentha spp. & hortelã; mentinha & 24086 & 15 & $\mathrm{E}$ & $\mathrm{HB} / \mathrm{P}$ \\
\hline Nepeta cataria L. & cidreira; melissa; cidró & 24087 & 4 & E & $\mathrm{HB} / \mathrm{P}$ \\
\hline Ocimum selloi Benth. & alfavaca & 24088 & 2 & $\mathrm{~N}$ & $\mathrm{SS} / \mathrm{P}$ \\
\hline Origanum majorana $\mathrm{L}$. & manjerona & 24089 & 7 & $\mathrm{E}$ & $\mathrm{HB} / \mathrm{P}$ \\
\hline Origanum vulgare $\mathrm{L}$. & orégano & 24090 & 1 & $\mathrm{E}$ & $\mathrm{HB} / \mathrm{P}$ \\
\hline Plectranthus barbatus Andrews & boldo-do-chile & 24091 & 1 & $\mathrm{E}$ & HB-SS/P \\
\hline Rosmarinus officinalis L. & alecrim & 24092 & 3 & $\mathrm{E}$ & $\mathrm{SS} / \mathrm{P}$ \\
\hline Salvia microphylla H.B.K. & melhoral; melhorar & 24093 & 6 & $\mathrm{E}$ & HB-SS/P \\
\hline Salvia officinalis $\mathrm{L}$. & sábia; sálvia & 24094 & 7 & $\mathrm{E}$ & $\mathrm{HB} / \mathrm{P}$ \\
\hline Stachys byzantina $\mathrm{K}$. Koch ex Scheele & pulmonária & 24095 & 6 & $\mathrm{E}$ & $\mathrm{HB} / \mathrm{P}$ \\
\hline \multicolumn{6}{|l|}{ Lauraceae } \\
\hline Laurus nobilis L. & louro & 24096 & 2 & $\mathrm{E}$ & SB-AR/P \\
\hline Persea americana Mill. & abacate & 24097 & 1 & $\mathrm{E}$ & $\mathrm{AR} / \mathrm{P}$ \\
\hline \multicolumn{6}{|l|}{ Liliaceae } \\
\hline Allium cepa $\mathrm{L}$. & cebola & 24098 & 1 & $\mathrm{E}$ & $\mathrm{HB} / \mathrm{P}$ \\
\hline Allium sativum L. & alho & 24099 & 1 & $\mathrm{E}$ & $\mathrm{HB} / \mathrm{P}$ \\
\hline Urginea maritima (L.) Baker & cebola-do-mar & 24100 & 4 & $\mathrm{E}$ & $\mathrm{HB} / \mathrm{P}$ \\
\hline
\end{tabular}


APPENDIX I (continuation)

\begin{tabular}{|c|c|c|c|c|c|}
\hline Botanical name & Common name & Voucher \# & $\mathrm{MF}$ & $\mathrm{N} / \mathrm{E}$ & Growing habit \\
\hline \multicolumn{6}{|l|}{ Lythraceae } \\
\hline Cuphea carthaginensis (Jacq.) Macbr. & sete-sangrias & 24101 & 3 & $\mathrm{~N}$ & $\mathrm{HB} / \mathrm{A}$ \\
\hline \multicolumn{6}{|l|}{ Malvaceae } \\
\hline Malva sylvestris L. & malva & 24102 & 9 & $\mathrm{E}$ & $\mathrm{HB} / \mathrm{B}$ or $\mathrm{P}$ \\
\hline Sida rhombifolia $\mathrm{L}$. & guanchuma & 24103 & 2 & $\mathrm{~N}$ & HB-SS/A or P \\
\hline \multicolumn{6}{|l|}{ Mimosaceae } \\
\hline Anadenanthera $s p$. & anjico & 24104 & 1 & $\mathrm{~N}$ & $\mathrm{AR} / \mathrm{P}$ \\
\hline \multicolumn{6}{|l|}{ Moraceae } \\
\hline Morus alba $\mathrm{L}$. & amora-branca & 24105 & 1 & $\mathrm{E}$ & SB-AR/P \\
\hline \multicolumn{6}{|l|}{ Myrtaceae } \\
\hline Campomanesia xanthocarpa O.Berg & guavirova; gaviroma; gabiroba & 24106 & 1 & $\mathrm{~N}$ & $\mathrm{AR} / \mathrm{P}$ \\
\hline Eucalyptus sp. & eucalipto & 24107 & 2 & $\mathrm{E}$ & $\mathrm{AR} / \mathrm{P}$ \\
\hline Eugenia involucrata DC. & cereja & 24108 & 1 & $\mathrm{~N}$ & $\mathrm{AR} / \mathrm{P}$ \\
\hline Eugenia uniflora $\mathrm{L}$. & pitanga & 24109 & 4 & $\mathrm{~N}$ & $\mathrm{AR} / \mathrm{P}$ \\
\hline Myrciaria cauliflora (DC.) Berg. & jaboticaba & 24110 & 1 & $\mathrm{~N}$ & $\mathrm{AR} / \mathrm{P}$ \\
\hline \multicolumn{6}{|l|}{ Papaveraceae } \\
\hline Argemone mexicana $\mathrm{L}$. & cardo-santo & 24111 & 1 & $\mathrm{E}$ & $\mathrm{HB} / \mathrm{A}$ \\
\hline \multicolumn{6}{|l|}{ Passifloraceae } \\
\hline Passiflora alata Dryander & maracujá & 24112 & 1 & $\mathrm{~N}$ & $\mathrm{PC} / \mathrm{P}$ \\
\hline \multicolumn{6}{|l|}{ Phytolaccaceae } \\
\hline Petiveria alliacea $\mathrm{L}$. & guiné & 24113 & 5 & $\mathrm{~N}$ & SS-SB/P \\
\hline \multicolumn{6}{|l|}{ Piperaceae } \\
\hline Piper mikanianum (Kunth) Steud. & pariparoba & 24114 & 3 & $\mathrm{~N}$ & SS-SB/P \\
\hline \multicolumn{6}{|l|}{ Plantaginaceae } \\
\hline Plantago australis Lam. & tansagem & 24115 & 9 & $\mathrm{~N}$ & $\mathrm{HB} / \mathrm{A}$ \\
\hline Plantago major $\mathrm{L}$. & tansagem & 24116 & 1 & $\mathrm{E}$ & HB/B-P \\
\hline \multicolumn{6}{|l|}{ Poaceae } \\
\hline Bambusa sp. & taquara & 24117 & 1 & $\mathrm{E}$ & $\mathrm{SB} / \mathrm{P}$ \\
\hline Cymbopogon citratus (DC.) Stapf & capim-limão; cidreira & 24118 & 4 & $\mathrm{E}$ & $\mathrm{HB} / \mathrm{P}$ \\
\hline \multicolumn{6}{|l|}{ Polygonaceae } \\
\hline Polygonum sp. & erva-de-bixo & 24119 & 3 & $\mathrm{E}$ & HB/A-P \\
\hline \multicolumn{6}{|l|}{ Polipodiaceae } \\
\hline Microgramma squamulosa (Kaulf.) Sota & sordinha & 24120 & 1 & $\mathrm{~N}$ & $\mathrm{PC} / \mathrm{HB} / \mathrm{P}$ \\
\hline \multicolumn{6}{|l|}{ Pteridaceae } \\
\hline Adianthum raddianum Presl. & avenca & 24121 & 4 & E & $\mathrm{HB} / \mathrm{P}$ \\
\hline Adiantopsis chlorophylla (Sw.) Fée & samambaia-roxa & 24122 & 1 & $\mathrm{E}$ & $\mathrm{HB} / \mathrm{P}$ \\
\hline \multicolumn{6}{|l|}{ Punicaceae } \\
\hline Punica granatum L. & romã & 24123 & 1 & E & SB-AR/P \\
\hline \multicolumn{6}{|l|}{ Rosaceae } \\
\hline Agrimonia eupatoria $\mathrm{L}$. & agrimonia & 24124 & 1 & $\mathrm{E}$ & $\mathrm{HB} / \mathrm{P}$ \\
\hline Eriobotrya japonica (Thunb.) Lindley & ameixeira-de-inverno; ameixa & 24125 & 3 & $\mathrm{E}$ & $\mathrm{AR} / \mathrm{P}$ \\
\hline Fragaria vesca $\mathrm{L}$. & morango & 24126 & 1 & $\mathrm{E}$ & $\mathrm{HB} / \mathrm{P}$ \\
\hline Prunus persica (L.) Batsch. & pêssego & 24127 & 1 & $\mathrm{E}$ & $\mathrm{AR} / \mathrm{P}$ \\
\hline Rosa sp. & rosa & 24128 & 1 & $\mathrm{E}$ & $\mathrm{SS} / \mathrm{P}$ \\
\hline Rubus brasiliensis Mart. & amora-branca & 24129 & 1 & $\mathrm{~N}$ & $\mathrm{SB} / \mathrm{P}$ \\
\hline \multicolumn{6}{|l|}{ Rutaceae } \\
\hline Ruta graveolens $\mathrm{L}$. & arruda & 24130 & 6 & $\mathrm{E}$ & $\mathrm{SS} / \mathrm{P}$ \\
\hline \multicolumn{6}{|l|}{ Simaroubaceae } \\
\hline Picrasma palo-amargo Speg. & pau-amargo & 24131 & 2 & $\mathrm{~N}$ & $\mathrm{AR} / \mathrm{P}$ \\
\hline
\end{tabular}


APPENDIX I (continuation)

\begin{tabular}{l|l|l|l|l|l}
\hline \multicolumn{1}{c|}{ Botanical name } & \multicolumn{1}{|c|}{ Common name } & Voucher \# & MF & N/E & Growing habit \\
\hline Solanaceae & & & & & \\
\hline Solanum licopersicum L. & folha-de-tomate & 24132 & 1 & $\mathrm{E}$ & HB/A-P \\
\hline Solanum sp. & erva-de-galinha & 24133 & 1 & $\mathrm{~N}$ & $\mathrm{SS} / \mathrm{A}-\mathrm{P}$ \\
\hline Urticaceae & & & & & \\
\hline Urera baccifera (L.) Gaud. & urtigão & 24134 & 1 & $\mathrm{~N}$ & $\mathrm{SB} / \mathrm{P}$ \\
\hline Verbenaceae & & & & & \\
\hline Stachytarpheta cayennensis (Rich.) Vahl & gervão & 24135 & 9 & $\mathrm{~N}$ & $\mathrm{SS} / \mathrm{P}$ \\
\hline Aloysia citriodora Palau & cidró & 24136 & 1 & $\mathrm{E}$ & $\mathrm{SB} / \mathrm{P}$ \\
\hline Lippia alba (Mill.) N.E.Br. & erva-cidreira; salva; sálvia & 24137 & 6 & $\mathrm{~N}$ & $\mathrm{SS} / \mathrm{P}$ \\
\hline Vitex megapotamica (Spreng.) Moldenke & tarumã & 24138 & 2 & $\mathrm{~N}$ & $\mathrm{SB} / \mathrm{P}$ \\
\hline Violaceae & & & & & \\
\hline Viola tricolor L. & violeta & 24139 & 3 & $\mathrm{E}$ & $\mathrm{HB} / \mathrm{P}$ \\
\hline Vitaceae & & & & & \\
\hline Vitis vinifera L. & parreira & 24140 & 1 & $\mathrm{E}$ & $\mathrm{PC} / \mathrm{P}$ \\
\hline Zingiberaceae & & & & & \\
\hline Zingiber officinalis Rosc. & gengibre & 24141 & 1 & $\mathrm{E}$ & $\mathrm{HB} / \mathrm{P}$ \\
\hline Alpinia zerumbet (Pers.) B.L. Burtt. and R.M.Sm. & nós-moscada & 24142 & 1 & $\mathrm{E}$ & $\mathrm{HB} / \mathrm{P}$ \\
\hline
\end{tabular}

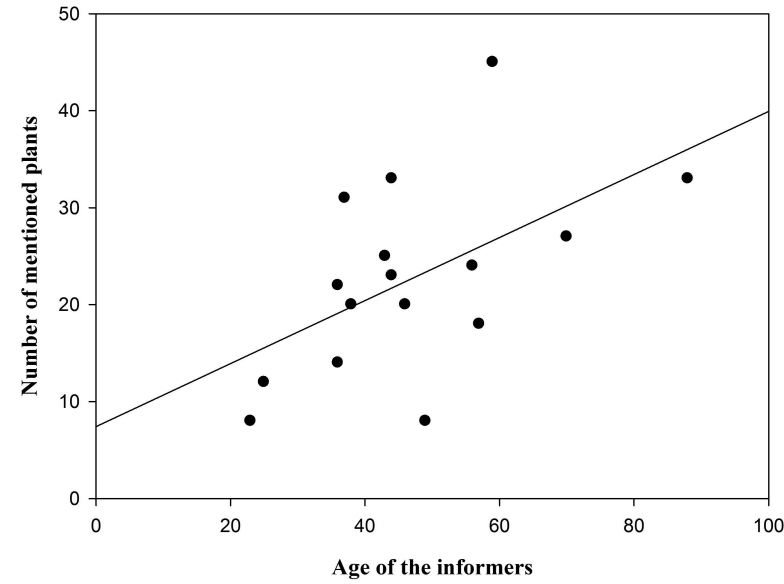

Fig. 3 - The influence of the informants' age on the number of plants mentioned during the ethnobotanical survey carried out in "Passo da Ilha" rural community, in the city of Pato Branco, Southern Brazil.

consumer's profile, some of the informers received financial support and orientation to start commercial cultivation of medicinal plants and spices.

\section{ACKNOWLEDGMENTS}

The authors would like to thank Secretaria de Educação Superior/Ministério da Educação (Sesu/MEC) and the program "Universidade Solidária" for the financial support for this project.

\section{RESUMO}

O objetivo deste trabalho foi resgatar e documentar o conhecimento popular referente às plantas medicinais utilizadas na comunidade rural do Passo da Ilha, em Pato Branco-PR $\left(26^{\circ} 11^{\prime} \mathrm{S}, 52^{\circ} 36^{\prime} \mathrm{W}\right.$ e $760 \mathrm{~m}$ de altitude). Optou-se por utilizar o modelo de entrevista estruturada com 16 informantes, os quais possuíam o conhecimento sobre o uso das plantas medicinais em suas famílias. O período da realização das entrevistas foi de outubro a dezembro de 2000. As plantas foram coletadas no campo, identificadas e "vouchers" foram depositados no Herbário "Irina Delanova De Gemtchjnicov" (BOTU) da Universidade Estadual Paulista, em Botucatu-SP. Foram identificadas 47 famílias botânicas e 114 espécies de plantas medicinais. Estas plantas foram indicadas para mais de 30 usos medicinais diferentes. A comunidade é composta na maioria por descendentes de europeus, justificando a presença de muitas plantas exóticas. O domínio do uso das plantas é feminino, porém o conhecimento é menor nas gerações mais novas, denotando um processo de erosão cultural.

Palavras-chave: erosão cultural, etnobotânica, plantas medicinais, conhecimento tradicional.

\section{REFERENCES}

Alexiades MN. 1996. Selected Guidelines for Ethnobotanical Research: A Field Manual. New York Botanical Garden, New York, 306 p. 
Amorozo MCM. 2002. Uso e diversidade de plantas medicinais em Santo Antonio do Leverger, MT, Brasil. Acta Bot Bras 16: 189-203.

Amorozo MCM. 2004. Pluralistic Medical Settings and Medicinal Plant use in Rural Communities, Mato Grosso, Brazil. J Ethnobiol 24: 139-161.

Begossi A, Hanazaki N, And Tamashiro JY. 2002. Medicinal Plants in the Atlantic Forest (Brazil): knowledge. Use and Conservation. Hum Ecol 30: 281-299.

Chrisman NJ. 1977. The Health Seeking Process: An Approach to The Natural History of Illness. Cult Med Psychiat 1: 351-377.

DAVIM RMB, Lima JFV, Silva RAR AND SILVA DAR. 2003. O Cuidado Materno: O Uso de Plantas Medicinais nas Doenças da Infância. Rev Tec Enferm 1: 292-296.

DORIGONi PA, GHEdini PC, Fróes LF, BAPTISTA KC, Ethur ABM, Baldisserotto B, Bürger ME, Almeida CE, Lopes AMV and ZÁchia RA. 2001. Levantamento de Dados Sobre Plantas Medicinais de Uso Popular no Município de São João do Polêsine, RS, Brasil. I - Relação Entre Enfermidades e Espécies Utilizadas. Rev bras plantas med 4: 69-79.

Elizabetsky E. 1991. Sociopolitical, Economical and Ethical Issues in Medicinal Plant Research. J Ethnopharmacol 32: 235-239.

ElizABETSKY E. 2000. Etnofarmacologia Como Ferramenta de Busca de Substâncias Ativas. In: Farmacognosia: da planta ao medicamento, SIMÕES CMO ET AL. (Eds), ed. da UFRGS/UFSC, Porto Alegre/Florianópolis, p. 87-100.

FARnSworth NR. 1988. Screening Plants for New Medicines. In: Wilson EO ANd Peter FM (Eds), Biodiversity. Nac Acad Press, Washington DC, USA, p. 83-97.

Farnsworth NR, Akerele O, Bingel AS, Soejarto DD AND Guo Z. 1985. Medicinal Plants in Therapy. Bull WHO 63: 965-981.

FRANCO EAP AND BARRos RFM. 2006. Uso e Diversidade de Plantas Medicinais no Quilombo Olho D'água dos Pires, Esperantina, Piauí. Rev bras plantas med 8: 78-88.

GARlet TMB AND IRGANG BE. 2001. Plantas Medicinais Utilizadas na Medicina Popular por Mulheres Trabalhadoras Rurais de Cruz Alta, Rio Grande do Sul, Brasil. Rev bras plantas med 4: 9-18.
HANAZAKI N, TAMAShIRO JY, LEITÃo-FILHO HF AND Begossi A. 2000. Diversity of Plants Use in Two Caiçara Communities from the Atlantic Forest Coast, Brazil. Biodivers Conserv 9: 597-615.

Heinrich M, Ankli A, Frei B, Weiman C And Sticher O. 1998. Medicinal Plants in Mexico: Healers' consensus and Cultural Importance. Soc Sci Med 47: 1859-1871.

LAIRD SA AND KATE KT. 2002. Biodiversity Prospecting: The Commercial Use of Genetic Resources and Best Practice in Benefit-sharing. In: LAIRD S (Ed), Biodiversity and Traditional knowledge: Equitable Partnerships in Practice. Earthscan Publications Ltd, London, p. $241-286$.

Marchese JA et AL. 2004. Perfil dos Consumidores de Plantas Medicinais e Condimentares do Município de Pato Branco (PR). Hortic Bras 22: 332-335.

Marodin SM AND BAPTISTA LR DE M. 2001. O Uso de Plantas com Fins Medicinais no Município de Dom Pedro Alcântara, Rio Grande do Sul, Brasil. Rev bras plantas med 4: 57-68.

Martin GJ. 1995. Etnobotánica: Manual de Métodos. Editorial Nordan-Comunidad, Montevideo.

MING LC. 1995. Coleta de Plantas Medicinais. In: Di STASI LC (Ed), Plantas Medicinais: Arte e Ciência/Um Guia de Plantas Medicinais. Editora da UNESP, São Paulo, SP, Brasil, p. 69-86.

Monteiro JM, Albuquerque UP, Lins-Neto EMF, ARAÚJo EL AND AMORIM ELC. 2006. Use Patterns and Knowledge of Medicinal Species Among Two Rural Communities in Brazil's Semi-Arid Northeastern Region. J Ethnopharmacol 105: 173-186.

MotomiYa AV DE A. 2004. Levantamento e Cultivo das Espécies de Plantas Medicinais Utilizadas em Cassilândia, MS. Paper presented at $2^{\text {nd }}$ Brazilian Meeting of University Extension. 12-15 September, Belo Horizonte, MG, Brasil.

Voltolini S. 1996. Retorno: Origens de Pato Branco. Artepress, Dois Vizinhos, 286 p. 